\title{
Effects of time-of-day on oxidative stress, cardiovascular parameters, biochemical markers, and hormonal response following level-1 Yo-Yo intermittent recovery test
}

\author{
K Aloui $^{1,2}$, S Abedelmalek ${ }^{3}$, H Chtourou ${ }^{1}$, DP Wong ${ }^{4}$, N Boussetta ${ }^{1}$, N Souissi ${ }^{1}$ \\ ${ }^{1}$ Research Laboratory "Sports Performance Optimization", National Center of Medicine and Science in Sports \\ (CNMSS), Tunis, Tunisia \\ ${ }^{2}$ Faculty of Sciences of Bizerte, University of Carthage, Bizerte, Tunisia \\ ${ }^{3}$ Department of Physiology and Functional Explorations, Faculty of Medicine of Sousse, University of Sousse, \\ Sousse, Tunisia \\ ${ }^{4}$ Human Performance Laboratory, Technological and Higher Education Institute of Hong Kong, Hong Kong, China
}

Received: April 18, 2016

Accepted: December 27, 2016

\begin{abstract}
The aim of this study was to investigate the effect of time-of-day on oxidative stress, cardiovascular parameters, muscle damage parameters, and hormonal responses following the level-1 Yo-Yo intermittent recovery test (YYIRT). A total of 11 healthy subjects performed an intermittent test (YYIRT) at two times-of-day (i.e., 07:00 $\mathrm{h}$ and 17:00 h), with a recovery period of $\geq 36 \mathrm{~h}$ in-between, in a randomized order. Blood samples were taken at the rest (baseline) and immediately (post-YYIRT) after the YYIRT for measuring oxidative stress, biochemical markers, and hormonal response. Data were statistically analyzed using one-way and two-way repeated measures ANOVA and Bonferroni test at $p<0.05$. Observed power $(\alpha=0.05)$ and partial eta-squared were used. Our results showed that oxygen uptake $\left(\mathrm{VO}_{2 \max }\right)$, maximal aerobic speed, and the total distance covered tended to be higher in the evening $(17: 00 \mathrm{~h})$. There was also a main effect of time-of-day for cortisol and testosterone concentration, which were higher after the YYIRT in the morning $(p<0.05)$. The heart rate peak and the rating of perceived exertion scales were lower in the morning $(p<0.05)$. However, the plasma glucose $(p<0.01)$, malondialdehyde, creatine kinase $(p<0.01)$, lactate dehydrogenase $(p<0.05)$, high-density lipoprotein $(p<0.01)$, total cholesterol $(p<0.01)$, and triglycerides $(p<0.05)$ were higher after the YYIRT in the evening. Low-density lipoprotein, systolic blood pressure, diastolic blood pressure, and lactate levels $(p>0.05)$ were similar for the morning and evening test. In conclusion, our findings suggest that aerobic performance presents diurnal variation with great result observed in the evening accompanied by an improvement of hormonal, metabolic, and oxidative responses. These data may help to guide athletes and coaches and contribute to public health recommendations on exercise and muscle damage particularly in the competitive periods.
\end{abstract}

Keywords: YYIRT, time-of-day, cardiovascular response, biochemical response, hormonal response, oxidative stress

\section{Introduction}

It is well established that both strenuous long-term and short-term anaerobic (repeated sprint test: $4 \times 15 \mathrm{~s}$, with 1-min rest intervals) exercises were reported to enhance the generation of

Corresponding author: Salma Abedelmalek, $\mathrm{PhD}$

Department of Physiology and Functional Explorations, Faculty of Medicine of Sousse, University of Sousse Ave Mohamed Karoui, 4002 Sousse, Tunisia

Phone: +216 22763272; Fax: +216 74226647; E-mails: s_abedelmalek@yahoo.fr; Maha.Daoud@isaas.rnu.tn 
reactive oxygen species (ROS) by cells, which may lead to oxidative stress and cellular damage (26). These ROS are considered as potent oxidative stress indicators in biological systems $(14,37)$. Coaches and physical trainers impose high-intensity training to evaluate and to improve players' performances particularly during the competitive period. The level-1 Yo-Yo intermittent recovery test (YYIRT) is the most common physical test used to evaluate the ability to perform high-intensity intermittent exercise. In fact, the physiological measurements showed that aerobic energy turnover reached maximal values and that the anaerobic energy system was greatly taxed at the end of the test. Indeed, the YYIRT leads to alterations in stress caused by free radical production $(22,32)$. Hence, elite athletes are more vulnerable to physical performance alteration than sedentary persons, it is also reflected by physiological responses due to stress caused by free radical production $(22,32)$.

On the other hand, considerable attention has been paid to chronobiological aspects of the antioxidant system (20) during exercise particularly the YYIRT (22). In addition, several studies have shown that aerobic performances are time-of-day dependent $(15,31)$, with higher values at the end of the evening around the time of peak of the circadian rhythm of body temperature $(13,15)$. Hammouda et al. $(23)$ have concluded that the circadian rhythm of core temperature is not the only cause of diurnal variation of performance. In this context, Hammouda et al. (18) showed that biological markers of muscle damage and antioxidant status were time-of-day dependent with higher values observed in the evening during shortterm maximal exercise in young trained subjects. Thus, many biochemical, physiological, hormonal, and immune parameters are affected by circadian rhythms $(1,12,20)$.

As a result, the diurnal variation of biological responses may perturb the interpretation of the diurnal variation of the performance observed following the YYIRT (18), although the exact mechanisms to explain these changes have not been delineated.

Numerous studies have proposed to explain the alterations of performance following intermittent exercise (YYIRT) not only by the relationship between the increase in muscle damage and oxidative stress but also by altered hormonal response $(15,31)$.

Furthermore, only Nogueira et al. (31) have reported the relationship between hormonal profiles and muscle damage in response to YYIRT. However, this study did not take the diurnal variation of oxidative stress into consideration in relation with hormonal parameters. There is also limited research connecting circadian variations in hormone levels with intermittent exercise.

Thus, muscle damage, oxidative stress, and hormonal response may occur following the YYIRT. In addition, the evaluation of oxidative stress by markers of protein and lipid oxidation (19) and the hormonal response may be useful to better assess and quantify muscle stress following the YYIRT particularly for coaches and athletes. Thus, it seems logical to evaluate the diurnal variation of both metabolic and hormonal responses during the YYIRT.

To the best of our knowledge, no study has investigated the diurnal variation effect on plasma levels of biomarkers of muscle damage and stress oxidative, and hormonal responses during intermittent exercise (YYIRT). Thus, coaches would consider the effect of metabolic and oxidative alterations on the period just before and during the competition. Therefore, in this work, we are committed to checking the effect of time-of-day on the selected blood marker of oxidative stress [malondialdehyde (MDA)], biochemical markers, muscle damage markers [i.e., lactate, lactate dehydrogenase (LDH), and creatine kinase (CK)], lipid profile [i.e., high-density lipoprotein (HDL), total cholesterol (TC), triglycerides (TG), and lowdensity lipoprotein (LDL)], and glucose (GLC), cardiovascular parameters [i.e., systolic 
blood pressure (SBP) and diastolic blood pressure (DBP)] and hormonal parameters [cortisol and testosterone (TE)] following the YYIRT.

We hypothesized, therefore, that the evening is the best time to perform intermittent exercise, which is associated with a clear variation of cardiovascular, hormonal, and oxidative responses as well as biochemical responses. Moreover, hormonal responses are related to muscle damage to explain the diurnal variation of the performance after the YYIRT.

\section{Materials and Methods}

\section{Participants}

A total of 11 healthy physical education students [mean \pm standard deviation (SD): age, $21.00 \pm 0.48$ years; height, $181.36 \pm 2.28 \mathrm{~cm}$; body weight, $72.75 \pm 1.79 \mathrm{~kg}$; body mass index (BMI), $22.15 \pm 0.54 \mathrm{~kg} / \mathrm{m}^{2}$ ] volunteered to participate in this study. After receiving a thorough explanation of the protocol, they gave written consent to participate in this study. This study protocol was in accordance with the Declaration of Helsinki for human experimentation and was approved by the University Ethics Committee. The participants were also selected based on their chronotype and on the basis of their answers to Horne and Ostberg (25) self-assessment questionnaire. They had an intermediate chronotype (i.e., sleep duration between 23:00 \pm 1:00 $\mathrm{h}$ and 07:00 \pm 1:00 h) and kept standard times for eating prior to the beginning of this study (breakfast at 07:00 \pm 1:00 h, lunch at 12:00 \pm 1:00 $\mathrm{h}$, and dinner at 20:00 \pm 1:00 h) (2). Subjects were non-smokers and did not consume nutritional supplements, caffeine, drugs, or alcoholic beverages. In addition, subjects suffering from diabetes mellitus, obesity, cardiovascular, pulmonary, or renal disorders were excluded from this study. Subjects were exercising for at least 4 days a week for an average of $2 \mathrm{~h}$ per day. Throughout the experimental period, participants were required to maintain their habitual physical activity and to avoid strenuous physical efforts $24 \mathrm{~h}$ before each test session.

\section{Study design}

The experimental procedure is illustrated in Fig. 1. Subjects performed YYIRT at two times-of-day (i.e., 07:00 $\mathrm{h}$ and 17:00 h) with a recovery period of $\geq 36 \mathrm{~h}$ in-between. At these
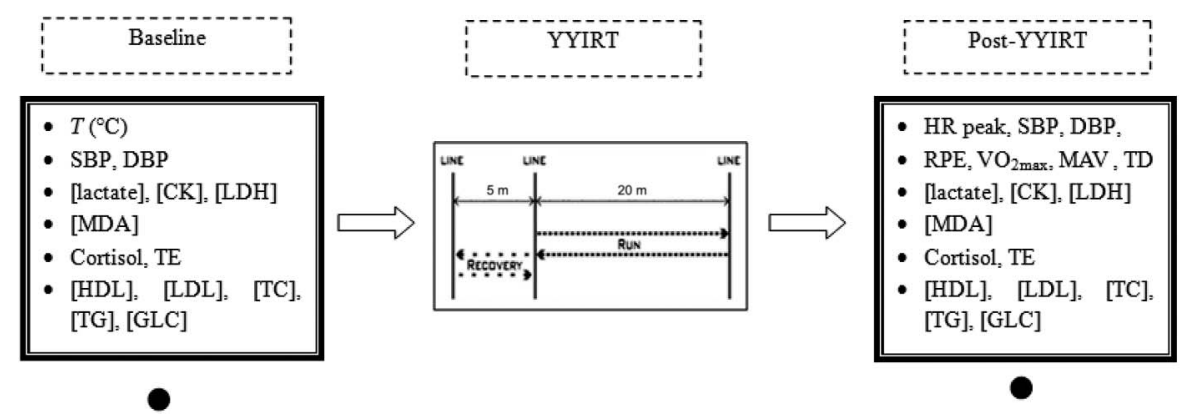

Fig. 1. Experimental protocol. $T\left({ }^{\circ} \mathrm{C}\right)$ : oral temperature; HR peak: heart rate peak; SBP: systolic blood pressure; DBP: diastolic blood pressure; [CK]: creatine kinase; [GLC]: glucose; [LDH]: lactate dehydrogenase; [MDA]: malondialdehyde; [HDL]: high-density lipoprotein; LDL: low-density lipoprotein; [TC]: total cholesterol; [TG]: triglycerides; TE: testosterone; YYIRT: Yo-Yo intermittent recovery test; TD: total distance covered during the YYIRT; RPE: rate of perceived exertion; $\mathrm{VO}_{2 \max }$ : maximal oxygen uptake; MAS: maximal aerobic speed; $\bullet$ : blood sample 
two time points, the YYIRT started with a measure of oral temperature, and the temperature was recorded using a digital clinical thermometer (Omron, Paris, France; accuracy $\pm 0.1{ }^{\circ} \mathrm{C}$ ) inserted sublingually for at least 3 min after a 15 -min rest in a seated position. To ensure a reliable measure of temperature, subjects were instructed not to talk or drink during measurement. The weight and body composition of subjects were then recorded using bioelectrical impedance scale to the nearest $0.1 \mathrm{~kg}$ (Tanita, Tokyo, Japan) calibrated in accordance with the manufacturer's guidelines by one trained technician. The BMI $\left(\mathrm{kg} / \mathrm{m}^{2}\right)$ was then calculated. Following this, duplicate measurements were taken with participants standing and wearing only briefs, as recommended by the guidelines. The average of these two measurements was used for the final analysis. The heart rate (HR) and blood pressure were monitored using Automatic Blood Pressure (BP W90, Microlife, Paris, France).

The YYIRT was performed at air temperature between 27 and $28{ }^{\circ} \mathrm{C}$ and with $53 \%-56 \%$ relative humidity.

\section{Exercise protocol}

The YYIRT. In brief, it consists of repeated $20-\mathrm{m}$ runs at a progressively increased speed controlled by audio bleeps from a CD player in a surface with no abstractions. Each running bout is interspersed by a 10-s active recovery period where the subject jogs around a marker placed $5 \mathrm{~m}$ behind the starting line (6). The test is stopped if the subject decides that he cannot follow the rhythm imposed or twice fails to reach the finishing line in time. The performance measured in this test is the total distance (TD). Participants are strongly encouraged verbally throughout the test. All subjects carried out a warm-up period consisting of the first four running bouts in the test (19). The participants perform the test in the same condition (i.e., comfortable sports shoes and appropriate sports clothing).

Rating of perceived exertion (RPE). Before performing exercise testing, the RPE (7) scales were explained to each participant by trained practitioners (Table I). Thereby, during exercise testing, overall perception of muscle fatigue (RPE) and physical stress was assessed. RPE is considered as a valid method to quantify training in response to high-intensity, intermittent exercise (34).

\section{Data analyses}

Blood samples were taken from the antecubital vein, sitting, before (baseline) and $3 \mathrm{~min}$ after (post-YYIRT) completing the YYIRT. The test venous blood samples were drawn into heparinized and ethylenediaminetetraacetic acid test tubes $(4 \mathrm{ml})$. The venous blood was centrifuged (for $20 \mathrm{~min}$ at 3,000 rpm at a room temperature) to separate erythrocytes from plasma and were frozen and stored at $-80{ }^{\circ} \mathrm{C}$ until analysis. The determination of muscle damage markers was performed by the diagnostic reagent (CK-NAC FS) CQN code: L1 in serum (DiaSys Diagnostic Systems Gmbh, Holzheim, Germany) for the CK levels. The method used is that described by the IFCC (International Federation of Clinical Chemistry): UV (ultra-violet) method. Sensitivity is $1 \mathrm{UL} / \mathrm{l}$. The concentrations of LDH were determined by the kinetic method of the French Society of Clinical Biology (SFBC) (Biomaghreb, Loos, France). The coefficient of variation is $5 \%$. In regard to the enzymatic GLC assay, it is carried out using a photometric enzyme assay in the presence of the enzymes such as glucose oxidase and peroxidase (GOD - PAP) (GLUCOSE; GOD-PAP, Biomagreb). The concentrations of HDL-cholesterol and LDL-cholesterol were determined by a colorimetric enzymatic assay: direct test in homogeneous phase (liquicolor) (Kit; HUMAN, Ref: 10084: Gesellschaft für 
Table I. The 15-grade scale for rating of perceived exertion (RPE scale) (7)

\begin{tabular}{|l|l|}
\hline Rating & \multicolumn{1}{|c|}{ Perceived exertion } \\
\hline 6 & No exertion \\
\hline 7 & Extremely light \\
\hline 8 & - \\
\hline 9 & Very light \\
\hline 10 & - \\
\hline 11 & Light \\
\hline 12 & - \\
\hline 13 & Somewhat hard \\
\hline 14 & - \\
\hline 15 & Hard \\
\hline 16 & - \\
\hline 17 & Very hard \\
\hline 18 & - \\
\hline 19 & Extremely hard \\
\hline 20 & Maximal exertion \\
\hline
\end{tabular}

Biochemica und Diagnostica mbH, Wiesbaden, Germany). Sensitivity is $1.1 \mathrm{mg} / \mathrm{dl}$ and the coefficient of variation is $1.25 \%$. TC assay was determined by the enzymatic method (CHOD PAP) (CHOLESTEROL; GHOD-PAP, Biomagreb) using the same protocol with sample incubation at $37{ }^{\circ} \mathrm{C}$ for $5 \mathrm{~min}$. The concentrations of TG were determined by the enzymatic method (GPO - PAP) (CHOLESTEROL; GPO-PAP, Biomagreb). The lactate concentration (LACT2-Lactate Gen.2; Roche Diagnostics GmbH, Indianapolis, IN, USA) was determined using an enzymatic/colorimetric method (Roche COBAS, Roche, Basel, Switzerland). The limit detection is $0.2 \mathrm{mmol} / 1(1.8 \mathrm{mg} / \mathrm{dl})$.

\section{Hormonal responses}

Cortisol and TE concentrations were analyzed by enzyme-linked fluorescent assay (VIDAS, BioMerieux, Lyon, France).

\section{MDA-thiobarbituric acid reactive substances (TBARS)}

The estimation of MDA was determined using a spectrophotometric method based on the reaction between MDA and thiobarbituric acid (TBA). In brief, $200 \mu \mathrm{l}$ of plasma was added to $200 \mu \mathrm{l}$ of $30 \%(\mathrm{w} / \mathrm{v})$ trichloroacetic acid (TCA) and the mixture was then centrifuged at 3,500 rpm for $10 \mathrm{~min}$. $200 \mu \mathrm{l}$ of the supernatant was added to $800 \mu \mathrm{l}$ of $0.4 \%(\mathrm{w} / \mathrm{v}) \mathrm{TBA}$ and the mixture was incubated in a shaking water bath at $90{ }^{\circ} \mathrm{C}$ for $10 \mathrm{~min}$ and then placed directly into ice water. The concentration of MDA (determined at $532 \mathrm{~nm}$ ) was expressed in micromole per liter of plasma using a molar extinction coefficient of $1.56 \times 105 \mathrm{M}^{-1} \mathrm{~cm}^{-1}$. 


\section{Statistical analysis}

All analyses were performed using STATISTICA Software (StatSoft, Paris, France). Data were reported as mean $\pm \mathrm{SD}$. Parametric tests were performed once the assumption of normality was confirmed using the Shapiro-Wilk W test. One-way analysis of variance (ANOVA) was used to compare the morning-evening differences between the anthropometric parameters, YYIRT parameters data (i.e., $\mathrm{VO}_{2 \max }$, TD covered during the YYIRT, HR peak, and the RPE scales), and the oral temperature $\left({ }^{\circ} \mathrm{C}\right)$. Cardiovascular parameters (i.e., SBP and DBP), biochemical markers, muscle damage markers (i.e., LDH and CK), lipid profile (i.e., HDL, TC, TG, LDL, and GLC), oxidative stress marker (MDA), and hormonal response (i.e., cortisol and TE) data were analyzed using a two-way repeated measures ANOVA [2 (time-of-day) $\times 2$ (points of measurement)]. The Bonferroni post hoc test was performed whenever significant effects or a significant interaction was found using ANOVA. To assess the data practical significance, effect sizes were calculated as partial eta-squared $\left(\eta_{p}^{2}\right)$. The most important observation is that observed power varies widely as a function of random sampling error in the observed effect sizes. Note also that the observed power at the extremes clearly distinguishes between $42 \%$ and $90 \%$. All of this led to conclude that the study has sufficient power to detect any significant effect even with a sample size of 11. Statistical significance was set at $p<0.05$.

\section{Results}

YYIRT performance, temperature, HR peak, and RPE scales

The mean values for TD, $\mathrm{VO}_{2 \max }$, maximal aerobic speed (MAS), RPE scales, and HR peak following the YYIRT in the morning and evening are displayed in Table II and the temperature $\left({ }^{\circ} \mathrm{C}\right)$ measurement is presented in Fig. 2 . The ANOVA revealed that core temperature and RPE values were significantly higher in the evening compared with the morning $(F=11.9$; $\eta_{p}^{2}=0.76 ; p<0.01$ and $F=17.55 ; \eta_{p}^{2}=0.53 ; p<0.05$, respectively). Likewise, a significant time-of-day effect $\left(F=48.57 ; \eta_{p}^{2}=0.34 ; p<0.05\right)$ for the $\mathrm{TD}, \mathrm{VO}_{2 \max }$, and MAS during the YYIRT was observed. In fact, the YYIRT performance (i.e., TD, $\mathrm{VO}_{2 \max }$, and MAS) fluctuated with higher values observed at 17:00 h $(p<0.05)$.

Moreover, the HR peak increased after the YYIRT in the evening $\left(F=95.9 ; \eta_{p}^{2}=0.84\right.$; $p<0.05)$ compared with the morning.

Table II. YYIRT parameters, RPE scales, and HR peak measured at the two times-of-day (mean \pm SD) during the YYIRT

\begin{tabular}{|l|c|c|c|}
\hline & $\mathbf{0 7 : 0 0} \mathbf{~}$ & $\mathbf{1 7 : 0 0} \mathbf{~}$ & $\boldsymbol{p}$ value \\
\hline $\mathrm{VO}_{2 \max }(\mathrm{ml} / \mathrm{min} / \mathrm{kg})$ & $47.44 \pm 0.45$ & $49.40 \pm 0.74^{*}$ & 0.04 \\
\hline $\mathrm{MAS}(\mathrm{km} / \mathrm{h})$ & $13.87 \pm 0.20$ & $14.56 \pm 0.28^{*}$ & 0.04 \\
\hline $\mathrm{TD}(\mathrm{m})$ & $890.90 \pm 76.29$ & $1,149.09 \pm 107.20^{*}$ & 0.04 \\
\hline $\mathrm{RPE}$ & $16.18 \pm 0.51$ & $17.09 \pm 0.25^{*}$ & 0.001 \\
\hline HR peak (beats/min) & $192.81 \pm 1.32$ & $196.63 \pm 0.87^{*}$ & 0.001 \\
\hline
\end{tabular}

*Significantly different from 07:00 h values 


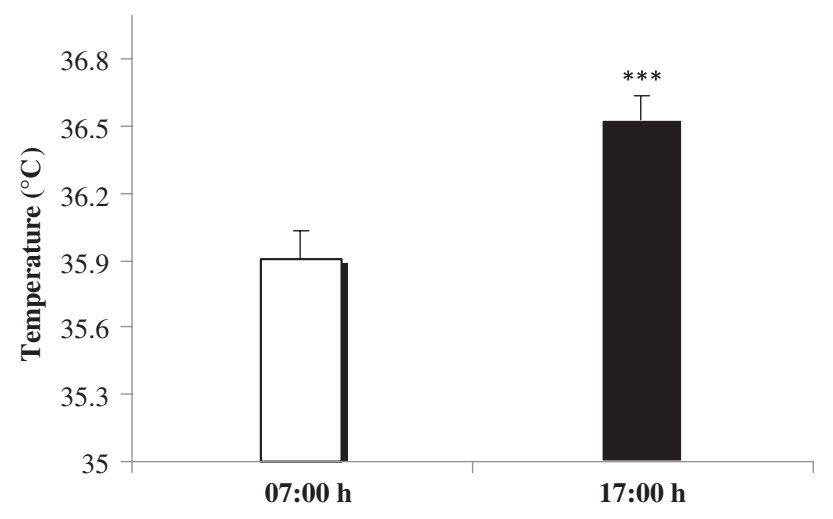

Fig. 2. Oral temperature $\left({ }^{\circ} \mathrm{C}\right)$ measured at 07:00 $\mathrm{h}$ and 17:00 $\mathrm{h}$ before the YYIRT. ***Significantly different from 17:00 h $(p<0.01)$

The cardiovascular parameters

The ANOVA showed a significant point of measurement effect for DBP $(F=17.38$; $\left.\eta_{p}^{2}=0.48 ; p<0.01\right)$ and SBP $\left(F=34.21 ; \eta_{p}^{2}=0.66 ; p<0.01\right)$ (Table III). However, no significant effect neither time-of-day nor interaction (time-of-day $\times$ points of measurement) was observed for SBP or DBP $(p>0.05)$.

Post hoc analysis showed that DBP and SBP levels registered presented a significant increase at post-YYIRT compared with baseline at both 07:00 $\mathrm{h}$ and 17:00 $\mathrm{h}$.

\section{MDA-TBARS}

Concerning MDA (Table III), the ANOVA revealed a significant time-of-day effect $(F=6.16$; $\left.\eta_{p}^{2}=0.87 ; p<0.05\right)$ and point of measurement effect $\left(F=6.55 ; \eta_{p}^{2}=0.56 ; p<0.05\right)$.

Table III. Cardiovascular parameters, muscle damage markers, hormonal responses, and MDA levels (mean \pm SD) measured before (baseline) and after (post-YYIRT) the YYIRT performed in the morning and the evening $(n=11)$

\begin{tabular}{|l|c|c|c|c|}
\hline \multirow{2}{*}{} & \multicolumn{2}{|c|}{ Baseline } & \multicolumn{2}{c|}{ Post-YYIRT } \\
\cline { 2 - 5 } & Morning & Evening & Morning & Evening \\
\hline DBP $(\mathrm{mmHg})$ & $81.90 \pm 2.64$ & $89.77 \pm 5.72$ & $97.72 \pm 2.53^{\dagger}$ & $92 \pm 3.09^{\dagger}$ \\
\hline SBP $(\mathrm{mmHg})$ & $134 \pm 2.33$ & $148 \pm 7.69$ & $159.81 \pm 2.53^{\dagger}$ & $161.63 \pm 6.68^{\dagger}$ \\
\hline Lactate $(\mathrm{mmol} / \mathrm{l})$ & $2.22 \pm 0.07$ & $2.32 \pm 0.06$ & $10.64 \pm 0.46^{\dagger}$ & $10.72 \pm 0.27^{\dagger}$ \\
\hline LDH $(\mathrm{UI} / \mathrm{l})$ & $264.54 \pm 24.27$ & $363.18 \pm 6.21$ & $420.90 \pm 28.61^{\dagger}$ & $458 \pm 23.30^{*, \dagger}$ \\
\hline $\mathrm{CK}(\mathrm{UI} / \mathrm{l})$ & $170.63 \pm 16.01$ & $222.27 \pm 1.81$ & $268.18 \pm 27.09^{\dagger}$ & $320 \pm 15.64^{*, \dagger}$ \\
\hline $\mathrm{Cortisol}(\mathrm{ng} / \mathrm{ml})$ & $139.24 \pm 9.77$ & $132.08 \pm 4.28$ & $155.33 \pm 7.97^{* *, \dagger}$ & $142.40 \pm 5.65^{\dagger}$ \\
\hline TE $(\mathrm{ng} / \mathrm{ml})$ & $7.08 \pm 0.37$ & $6.64 \pm 0.18$ & $7.04 \pm 0.25^{* *, \dagger}$ & $5.95 \pm 0.41^{\dagger}$ \\
\hline MDA $(\mu \mathrm{mol} / \mathrm{l})$ & $0.93 \pm 0.02$ & $1.02 \pm 0.05$ & $1.02 \pm 0.02^{\dagger}$ & $1.09 \pm 0.03^{*}{ }^{\dagger}$ \\
\hline
\end{tabular}

DBP: diastolic blood pressure; SBP: systolic blood pressure; LDH: lactate dehydrogenase; CK: creatine kinase; TE: testosterone; MDA: malondialdehyde.

*Main effect of time-of-day significantly lower in the morning than in the evening $(p<0.035)$.

**Main effect of time-of-day significantly higher in the morning than in the evening $(p<0.01)$.

${ }^{\dagger}$ Significantly different from baseline values $(p<0.001)$ 
However, no significant interaction (time-of-day $\times$ points of measurement) effect $(F=0.21$; $\left.\eta_{p}^{2}=0.03 ; p>0.05\right)$ was observed. The post hoc analysis showed that the MDA levels following the YYIRT presented a significant increase versus baseline at 07:00 $\mathrm{h}$ and 17:00 $\mathrm{h}$ $(p<0.05)$. Likewise, MDA concentrations were significantly higher in the evening in comparison with the morning after the YYIRT at rest $(p<0.05)$. In addition, the variation percentage induced by the YYIRT was significantly higher in the morning $(p<0.05)$.

\section{Markers of muscle damage}

The ANOVA showed a significant point of measurement effect $\left(F=33.88 ; \eta_{p}^{2}=0.80\right.$; $p<0.01)$ for the LDH concentrations, $\left(F=33.05 ; \eta_{p}^{2}=0.5 ; p<0.01\right)$ for the CK levels, and $\left(F=1,060.83 ; \eta_{p}^{2}=0.76 ; p<0.01\right)$ for the lactate levels observed after the YYIRT (Table III). Likewise, a significant time-of-day effect $\left(F=9.37 ; \eta_{p}^{2}=0.37 ; p<0.05\right)$ and $\left(F=11.21 ; \eta_{p}^{2}=0.61 ; p<0.01\right)$ was shown for LDH and CK levels, respectively. However, no significant time-of-day effect $\left(F=0.091 ; \eta_{p}^{2}=0.001 ; p>0.05\right)$ and interaction (time-of-day $\times$ points of measurement) for plasma concentrations of lactate, $\mathrm{LDH}$, and $\mathrm{CK}$ were observed.

The post hoc analysis revealed that lactate levels, similarly to the LDH and the CK levels presented a significant increase after the YYIRT compared to rest at 07:00 h and 17:00 $\mathrm{h}$ $(p<0.05)$. Moreover, the plasma concentrations of LDH and CK rise in the evening compared with those in the morning $(p<0.05)$. In addition, the variation percentage induced by the YYIRT was significantly higher in the morning $(p<0.05)$. Nevertheless, lactate levels did not follow a diurnal variation.

\section{The hormonal responses}

The ANOVA revealed a significant increase in the cortisol levels induced by exercise $\left(F=19.18 ; \eta_{p}^{2}=0.69 ; p<0.01\right)$ and in the TE levels $\left(F=27.20 ; \eta_{p}^{2}=0.78 ; p<0.01\right)$ (Table III). Furthermore, statistical analysis showed a significant time-of-day effect $\left(F=4.96 ; \eta_{p}^{2}=0.34 ; p<0.05\right)$ and $\left(F=5.90 ; \eta_{p}^{2}=0.35 ; p<0.05\right)$ for the cortisol and TE levels, respectively. However, the interaction (time-of-day $\times$ points of measurement) for the concentrations of cortisol $\left(F=1.29 ; \eta_{p}^{2}=0.02 ; p>0.05\right)$ and TE $\left(F=0.41 ; \eta_{p}^{2}=0.04\right.$; $p>0.05)$ was not significant.

Post hoc analysis indicated that the cortisol and TE levels registered presented a significant increase at post-YYIRT compared to rest at 07:00 $\mathrm{h}$ and 17:00 h. Likewise, these hormones were higher in the morning than in the evening at rest $(p<0.05)$. In addition, the percentage of variation of TE levels induced by YYIRT was significantly higher in the evening $(p<0.05)$. The normalized cortisol response to the exercise (post-YYIRT vs. baseline) was significantly higher in the morning than in the evening (Fig. 3A).

\section{The biochemical markers}

The ANOVA showed that exercise was associated with an increase in the LDL-cholesterol $\left(F=5.55 ; \eta_{p}^{2}=0.35 ; p<0.05\right)$, the HDL-cholesterol $\left(F=43.92 ; \eta_{p}^{2}=0.81 ; p<0.01\right)$, the TC $\left(F=41.28 ; \eta_{p}^{2}=0.80 ; p<0.01\right)$, the TG $\left(F=11.62 ; \eta_{p}^{2}=0.53 ; p<0.01\right)$, and the GLC $\left(F=55 ; \eta_{p}^{2}=0.34 ; p<0.01\right)$ levels after the YYIRT at 07:00 $\mathrm{h}$ and 17:00 $\mathrm{h}$ (Table IV). Moreover, the ANOVA revealed a significant increase in the $\operatorname{GLC}(F=13.74 ; p<0.01)$, HDL-cholesterol $\left(F=107.18 ; \eta_{p}^{2}=0.91 ; p<0.01\right)$, the TC $\left(F=11.40 ; \eta_{p}^{2}=0.53 ; p<0.01\right)$, and the TG $\left(F=9.45 ; \eta_{p}^{2}=0.48 ; p<0.05\right)$ levels in the evening, whereas LDL-cholesterol $\left(F=0.65 ; \eta_{p}^{2}=0.06 ; p>0.05\right)$ did not show any diurnal variation. Likewise, the interaction 

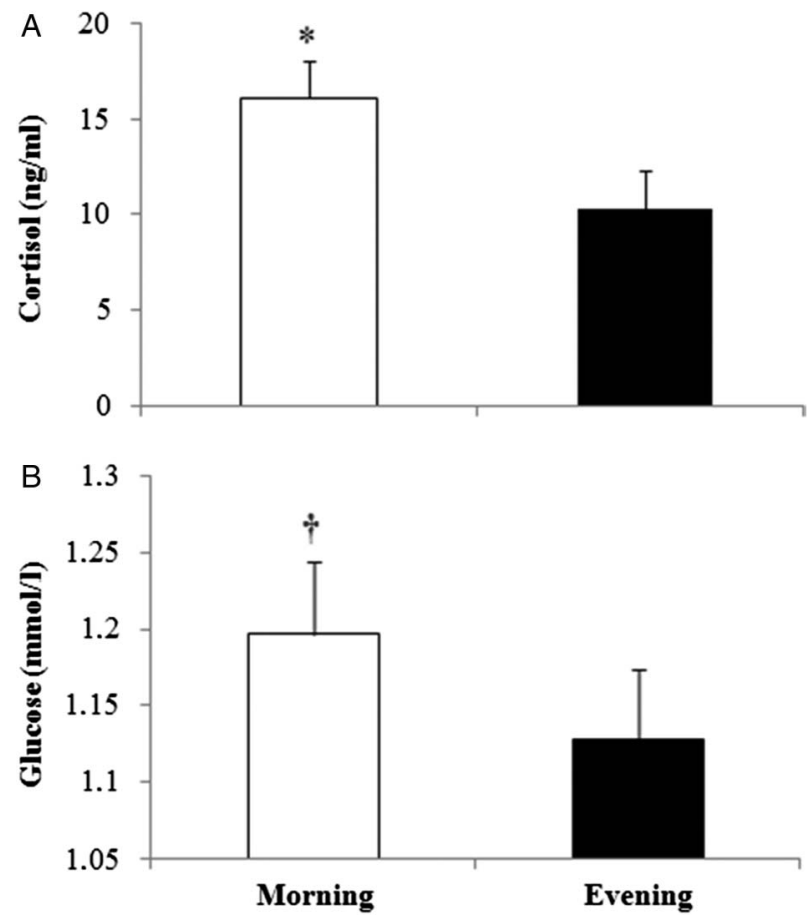

Fig. 3. Mean and SD for cortisol (A) and glucose $(\mathrm{B})$ response to the exercise (post-YYIRT vs. baseline). *Significantly higher in the morning than in the evening $(p<0.05)$.

${ }^{\dagger}$ Tendency to be higher in the morning than in the evening $(p<0.01)$

Table IV. Biochemical measurement (mean $\pm \mathrm{SD}$ ) measured before (baseline) and after (post-YYIRT) the YYIRT performed in the morning and the evening $(n=11)$

\begin{tabular}{|l|c|c|c|c|}
\hline \multirow{2}{*}{} & \multicolumn{2}{|c|}{ Baseline } & \multicolumn{2}{c|}{ Post-YYIRT } \\
\cline { 2 - 5 } & Morning & Evening & Morning & Evening \\
\hline LDL (mmol/l) & $0.98 \pm 0.11$ & $0.97 \pm 0.03$ & $1.21 \pm 0.05^{\dagger}$ & $1.12 \pm 0.03^{\dagger}$ \\
\hline HDL (mmol/l) & $0.76 \pm 0.03$ & $0.97 \pm 0.04$ & $1.18 \pm 0.07^{\dagger}$ & $1.40 \pm 0.07^{*}$, \\
\hline TC (mmol/l) & $2.92 \pm 0.16$ & $3.21 \pm 0.11$ & $3.83 \pm 0.15^{\dagger}$ & $4.07 \pm 0.15^{*, \dagger}$ \\
\hline TG (mmol/1) & $0.88 \pm 0.06$ & $1.05 \pm 0.06$ & $1.31 \pm 0.15^{\dagger}$ & $1.47 \pm 0.06^{*, \dagger}$ \\
\hline GLC (mmol/l) & $4.19 \pm 0.11$ & $4.81 \pm 0.09$ & $5.63 \pm 0.11^{\dagger}$ & $5.69 \pm 0.12^{*, \dagger}$ \\
\hline
\end{tabular}

LDL: low-density lipoprotein; HDL: high-density lipoprotein; TC: total cholesterol; TG: triglycerides; GLC: blood glucose.

*Main effect of time-of-day significantly lower in the morning than in the evening $(p<0.05)$.

${ }^{\dagger}$ Significantly different from baseline values $(p<0.001)$

(time-of-day $\times$ points of measurement) for plasma concentrations of TC $(F=0.01$; $\left.\eta_{p}^{2}=0.001 ; p>0.05\right), \mathrm{TG}\left(F=0.01 ; \eta_{p}^{2}=0.001 ; p>0.05\right)$, HDL $\left(F=0.03 ; \eta_{p}^{2}=0.003\right.$; $p>0.05)$, and LDL $\left(F=0.29 ; \eta_{p}^{2}=0.02 ; p>0.05\right)$ were not significant. However, a significant interaction (time-of-day $\times$ points of measurement) for GLC levels $(F=7.68$; 
$p<0.05)$ was observed. The normalized GLC response to the exercise tended also to be higher in the morning than in the evening (Fig. 3B).

\section{Discussion}

The main purpose of this study was to determine the effect of time-of-day on performance, energy system contribution, and metabolic, hormonal, and oxidative stress responses during YYIRT. The main findings of this study were: (a) a better performance was found in the evening than in the morning with an increased in RPE and HR peak; (b) cortisol and TE levels decreased in the evening and increased after the YYIRT. The GLC levels were lower in the morning and higher in response to YYIRT; (c) biochemical markers, muscle damage markers (i.e., LDH and CK), lipid profile (i.e., HDL, TC, and TG), and MDA levels were higher in the evening and increased after the YYIRT; (d) cardiovascular parameters (i.e., SBP and DBP) increased after the YYIRT.

In this study, our data showed that the performance was affected by the time-of-day during YYIRT, with improvement in the evening compared with the morning. Therefore, the diurnal variation of performance was confirmed, but the reason of the varied diurnal performance has remained unclear. Possible explanation may be that this improvement in performance in the evening might be associated with energy system distribution during the YYIRT test (19), an intermittent cycling exercise (36), repeated-sprint test (33), and $5 \times 6 \mathrm{~s}$ repeated sprint cycling (21). These results suggest that this improvement in the evening is not caused by the alteration in a unique energy system (i.e., aerobic or anaerobic) $(15,17,19)$. Instead, the enhancement of performance in the evening is due to a concomitant and maintained increase in aerobic and anaerobic contributions throughout the trial $(15,19)$. In the future, it would be interesting to investigate the concomitant effect of anaerobic exercise and time-of-day on biochemical markers, hormonal, and oxidative responses. Another explanation can elucidate, that, at least in part, the improvement of the performance is due to the peak of temperature observed generally in the evening with an increase in RPE scores. Furthermore, this raise of performance lead a potent change of the metabolic responses accompanied with an increase in the HR and blood pressure (SBP and DBP). These results are in accordance with previous reports $(13,18,27)$. However, Hammouda et al. (19) concluded that the circadian rhythm of core temperature is not the only cause of diurnal variation of performance. Exercise is also associated with a change in the lipid profile (4) which is an important indicator of cardiovascular health (19). Our results indicated that LDL, HDL, TC, and TG levels increased significantly during the YYIRT with higher values observed in the evening except for the LDL levels which did not fluctuate with the timeof-day. Therefore, the higher biochemical responses observed in the evening could explain, partially, the greater performance and metabolic demand at this time-of-day. The study of Hammouda et al. (19) indicated that the intensity of YYIRT is sufficient to increase biochemical parameters. Furthermore, this increase in lipid profile reflects the mobilization of purine cycle following this type of exercise. However, the exact mechanisms for this improvement have not been elucidated to date.

Thus, the diurnal variation of hormonal response can explain this improvement in relation with biochemical responses, particularly in trained subjects (35). In fact, athletes are more vulnerable concerning the alterations of biological parameters related to the biological body clock. As a result, a significant change in hormonal and metabolic responses between the parts of the day (i.e., morning and evening) can explain the improvement of performance 
observed in this study. In fact, cortisol levels were significantly higher in the morning compared with the evening $(3,21,30,31)$. This elevated cortisol at $07: 00 \mathrm{~h}$ caused by the YYIRT is indicative of an increased physiological stress, and it would be expected to elevate plasma GLC (15). Previous studies showed that the cortisol has an immune-suppressor effect $(2,16)$. We hypothesize therefore that elevated GLC concentrations in the morning after the exercise (with a percentage of variation $25.64 \%$ ) may not create an optimal metabolic milieu to meet the best performance. These findings suggest a possible link between the diurnal variation of metabolic as well as hormonal responses and the related pattern of YYIRT performances. Moreover, TE presents a biorhythmic effect with the highest values in the morning and the lowest in the evening. However, the higher levels of TE induced by YYIRT $(15.51 \%$ at $17: 00 \mathrm{~h}$ vs. $6.24 \%$ at $07: 00 \mathrm{~h})$ play a key role in the gaining of muscle; this is another reason why intermittent exercises should be performed in the evening.

Furthermore, it was demonstrated that physical exercise is characterized by an increase in the volume of oxygen consumed $(8,10)$ which leads to an increase in ROS production and causes oxidative stress. As a result, our data showed a significant increase in MDA immediately after the YYIRT. The increase is probably caused by the repeated ischemiareperfusion-like state following YYIRT, while the second is dependent on inflammation at the damaged site (1). This study demonstrates that oxidative stress is dependent on the time-of-day in trained subjects. Possible explanation was related to the production of pro-inflammatory cytokines in the skeletal muscle such us the interleukin 6 (IL-6) in response to exercise $(1,28)$. Unfortunately, plasma concentrations of IL-6 were not measured in this study.

In addition, Bouzid et al. (8) have demonstrated that there is a increase in lipid peroxidation level following acute exercise due to a higher production of ROS during and/ or following exercise and a positive correlation with markers of muscle damage. Our results illustrate that $\mathrm{CK}(36.37 \%$ at $07: 00 \mathrm{~h}$ vs. $30.53 \%$ at $17: 00 \mathrm{~h})$ and $\mathrm{LDH}(37.17 \%$ at 07:00 h vs. $20.7 \%$ at $17: 00 \mathrm{~h}$ ) levels induced by the YYIRT are significantly higher in the morning compared with the evening. These markers are essentially indicative of muscle damage which induces micro-lesions $(5,11)$ in the active muscle and decrease in the performance in the morning $(9,29)$. Thus, the increase in CK levels indicates that the athlete is more vulnerable to injury and muscle damage caused by an intense physical activity.

As a consequence, biochemical variables (i.e., $\mathrm{CK}$ ) and hormonal variables (i.e., TE and cortisol) are used to monitor muscle damage and disturbance in the hypothalamus-pituitaryadrenal and hypothalamus-pituitary-gonadal axis, respectively; all of them being indicators of fatigue accumulation (31). Thus, CK, TE, and cortisol are potential indicators to be used for monitoring disturbances caused by fatigue accumulation in response to the YYIRT. Therefore, it can be said that intermittent exercise is best to perform in the evening.

The present results show that lactate levels increased significantly after the YYIRT. Previous research has reported similar results and suggested that increased blood lactate concentrations after exercise caused alteration concerning red blood cell deformability and rigidity $(15,20,24)$. In accordance with Fernandes et al. (15), we have shown that lactate levels were not affected by the time-of-day. However, Racinais et al. (33) and Hammouda et al. (20) have demonstrated that lactate levels are higher in the evening after the exercise; therefore, it seems that athletes are more disposed to inflammation and free radical damage when exercise is done at this time. The conflicting findings may be attributed to the differences in the intensity and duration of exercise and the selected population. 


\section{Strengths and limitations}

To our knowledge, this is the first study to investigate the diurnal variation effect on plasma levels of hormones and stress-induced oxidative response during intermittent exercise. Moreover, results from the field of diurnal variation of the exercise and it's relation with stress and hormone response may help to guide athletes and contribute to public health recommendations on exercise and muscle damage. Furthermore, results can be monitored by the athlete, the coach, and the supporting staff to better plan training cycles throughout the training season and competition period. In addition, all references used in this study are recent. Likewise, this study has a limited number of participants and we did not use professional trained subjects. Furthermore, a limited number of samples are taken in this study. It is suggested to make a longer follow-up and take samples later after the exercise to examine the diurnal variation of oxidative stress, muscle damage, hormonal and cardiovascular parameters in the recovery period. Future studies should focus upon other markers of oxidative stress (i.e., carbonylated protein, glutathione, total antioxidant status, etc.).

\section{Conclusion}

In summary, we conclude that performance was impaired in the morning compared with that in the evening, associated with a patent variation of cardiovascular, hormonal, oxidative response as well as biochemical measures. Morning exercise was performed in a less favorable, hormonal, and metabolic milieu (i.e., elevated TE and cortisol), combined with an exacerbated plasma GLC response to the exercise. Thus, athletes and coaches should take into consideration the metabolic, hormonal, and oxidative alterations in the morning in comparison with the evening, in particular when planning the trainings for the period just before and during the competition.

\section{Acknowledgements}

The authors would like to thank Dr. Zeher Ben Jmaa and Dr. Fatma Ayadi (Biochemical Laboratory, Habib Bourguiba Hospital, Sfax, Tunisia) for their help in the realization of the Comet Test. This research received no specific grant from any funding agency in the public, commercial, or not-for-profit sectors.

\section{Conflict of interest}

The authors declare no conflict of interest.

$\begin{array}{ll}\text { Abbreviations } \\ \text { CK } & \text { : creatine kinase } \\ \text { DBP } & \text { : diastolic blood pressure } \\ \text { HDL } & \text { : high-density lipoprotein } \\ \text { HR } & \text { : heart rate } \\ \text { LDH } & : \text { lactate dehydrogenase } \\ \text { LDL } & : \text { low-density lipoprotein } \\ \text { MDA } & \text { : malondialdehyde } \\ \text { SBP } & \text { : systolic blood pressure } \\ \text { TC } & \text { : total cholesterol } \\ \text { TD } & \text { : total distance }\end{array}$


TG : triglycerides

TE : testosterone

MAS : maximal aerobic speed

YYIRT : Yo-Yo intermittent recovery test

\section{REFERENCES}

1. Abedelmalek S, Chtourou H, Aloui A, Aouichaoui C, Souissi N, Tabka Z: Effect of time of day and partial sleep deprivation on plasma concentrations of IL-6 during a short-term maximal performance. Eur. J. Appl. Physiol. 113(1), 241-248 (2013)

2. Abedelmalek S, Chtourou H, Souissi N, Tabka Z: Caloric restriction effect on proinflammatory cytokines, growth hormone, and steroid hormone concentrations during exercise in judokas. Oxid. Med. Cell. Longev. 2015, 809492 (2015)

3. Ahmed AM: Role of testosterone in glucose homeostasis in immobilization stressed rats. J. Am. Sci. 8(1), 628-638 (2012)

4. Awobajo FO, Olawale OA, Bassey S: Changes in blood glucose, lipid profile and antioxidant activities in trained and untrained adult male subjects during programmed exercise on the treadmill. Nig. Q. J. Hosp. Med. 23(2), 117-124 (2013)

5. Baird MF, Graham SM, Baker JS, Bickerstaff GF: Creatine-kinase- and exercise-related muscle damage implications for muscle performance and recovery. J. Nutr. Metab. 2012, 960363 (2012)

6. Bendiksen M, Bischoff R, Randers MB, Mohr M, Rollo I, Suetta C, Bangsbo J, Krustrup P: The Copenhagen Soccer Test: physiological response and fatigue development. Med. Sci. Sports Exerc. 44(8), 1595-1603 (2012)

7. Borg GA: Psychophysical bases of perceived exertion. Med. Sci. Sports Exerc. 14(5), 377-381 (1982)

8. Bouzid MA, Hammouda O, Matran R, Robin S, Fabre C: Changes in oxidative stress markers and biological markers of muscle injury with aging at rest and in response to an exhaustive exercise. PLoS One 9(3), e90420 (2014)

9. Brancaccio P, Lippi G, Maffulli N: Biochemical markers of muscular damage. Clin. Chem. Lab. Med. 48(6), 757-767 (2010)

10. Cardoso AM, Bagatini MD, Roth MA, Martins CC, Rezer JF, Mello FF, Lopes LF, Morsch VM, Schetinger MR: Acute effects of resistance exercise and intermittent intense aerobic exercise on blood cell count and oxidative stress in trained middle-aged women. Braz. J. Med. Biol. Res. 45(12), 1172-1182 (2012)

11. Ceci R, Valls MRB, Duranti G, Dimauro I, Quaranta F, Pittaluga M, Sabatini S, Caserotti P, Parisi P, Parisi A, Caporossi D: Oxidative stress responses to a graded maximal exercise test in older adults following explosivetype resistance training. Redox Biol. 2, 65-72 (2014)

12. Chin CY, Chow GCC, Hung KC, Kam LH, Chan KC, Mok YT, Cheng NM: The diurnal variation on cardiovascular endurance performance of secondary school athlete students. Asian J. Sports Med. 6(2), e22697 (2015)

13. Chtourou H, Hammouda O, Souissi H, Chamari K, Chaouachi A, Souissi N: Diurnal variations in physical performances related to football in young soccer players. Asian J. Sports Med. 3(3), 139-144 (2012)

14. El Abed K, Rebai H, Bloomer RJ, Trabelsi K, Masmoudi L, Zbidi A, Sahnoun Z, Hakim A, Tabka Z: Antioxidant status and oxidative stress at rest and in response to acute exercise in judokas and sedentary men. J. Strength Cond. Res. 25(9), 2400-2409 (2011)

15. Fernandes AL, Lopes-Silva JP, Bertuzzi R, Casarini DE, Arita DY, Bishop DJ, Lima-Silva AE: Effect of time of day on performance, hormonal and metabolic response during a 1000-M cycling time trial. PLoS One 9(10), e109954 (2014)

16. Gaysina D, Gardner MP, Richards M, Ben-Shlomo Y: Cortisol and cognitive function in midlife: the role of childhood cognition and educational attainment. Psychoneuroendocrinology 47, 189-198 (2014)

17. Giacomoni M, Billaut F, Falgairette G: Effects of the time of day on repeated all-out cycle performance and short-term recovery patterns. Int. J. Sports Med. 27, 468-474 (2006)

18. Hammouda O, Chahed H, Chtourou H, Ferchichi S, Miled A, Souissi N: Morning-to-evening difference of biomarkers of muscle injury and antioxidant status in young trained soccer players. Biol. Rhythm Res. 43(4), 431-438 (2012)

19. Hammouda O, Chtourou H, Aloui A, Chahed H, Kallel C, Miled A, Chamari K, Chaouachi A, Souissi N: Concomitant effects of Ramadan fasting and time-of-day on apolipoprotein AI, B, Lp-a and homocysteine responses during aerobic exercise in Tunisian soccer players. PLoS One 8(11), e79873 (2013) 
20. Hammouda O, Chtourou H, Chahed H, Ferchichi S, Chaouachi A, Kallel C, Miled A, Chamari K, Souissi N: High intensity exercise affects diurnal variation of some biological markers in trained subjects. Int. J. Sports Med. 33(11), 886-891 (2012)

21. Hammouda O, Chtourou H, Chahed H, Ferchichi S, Kallel C, Miled A, Chamari K, Souissi N: Diurnal variations of plasma homocysteine, total antioxidant status, and biological markers of muscle injury during repeated sprint: effect on performance and muscle fatigue - a pilot study. Chronobiol. Int. 28(10), 958-967 (2011)

22. Hammouda O, Chtourou H, Chaouachi A, Chahed H, Ferchichi S, Kallel C, Chamari K, Souissi N: Effect of short-term maximal exercise on biochemical markers of muscle damage, total antioxidant status, and homocysteine levels in football players. Asian J. Sports Med. 3(4), 239-246 (2012)

23. Hammouda O, Chtourou H, Chaouachi A, Chahed H, Zarrouk, N, Miled A, Chamari K, Souissi N: Biochemical responses to level-1 Yo-Yo intermittent recovery test in young Tunisian football players. Asian J. Sports Med. 4(1), 23-28 (2013)

24. Hayes LD, Bickerstaff GF, Baker JS: Interactions of cortisol, testosterone, and resistance training: influence of circadian rhythms. Chronobiol. Int. 27(4), 675-705 (2010)

25. Horne JA, Ostberg O: A self-assessment questionnaire to determine morningness-eveningness in human circadian rhythms. Int. J. Chronobiol. 4(2), 97-110 (1976)

26. Jówko E, Długołęcka B, Makaruk B, Cieśliński I: The effect of green tea extract supplementation on exerciseinduced oxidative stress parameters in male sprinters. Eur. J. Nutr. 54(5), 783-791 (2015)

27. Lakin, R, Notarius C, Thomas S, Goodman J: Effects of moderate-intensity aerobic cycling and swim exercise on post-exertional blood pressure in healthy young untrained and triathlon-trained men and women. Clin. Sci. (Lond.) 125(12), 543-553 (2013)

28. Main LC, Dawson B, Heel K, Grove JR, Landers GJ, Goodman C: Relationship between inflammatory cytokines and self-report measures of training overload. Res. Sports Med. 18(2), 127-139 (2010)

29. Mohebbi H, Azizi M: Maximal fat oxidation at the different exercise intensity in obese and normal weight men in the morning and evening. J. Human Sport Exerc. 6(1), 49-58 (2011)

30. Mora-Rodríguez R, Pallarés JG, López-Samanes Á, Ortega JF, Fernández-Elías VE: Caffeine ingestion reverses the circadian rhythm effects on neuromuscular performance in highly resistance-trained men. PLoS One 7(4), e33807 (2012)

31. Nogueira FDA, de Freitas VH, Nogueira RA, Miloski B, Werneck FZ, Bara-Filho MG: Improvement of physical performance, hormonal profile, recovery-stress balance and increase of muscle damage in a specific futsal preseason planning. Rev. Andal. Med. Deporte (in press), doi:10.1016/j.ramd.2015.11.008

32. Prahalathan P, Saravanakumar M, Raja B: The flavonoid morin restores blood pressure and lipid metabolism in DOCA-salt hypertensive rats. Redox Rep. 17(4), 167-175 (2012)

33. Racinais S, Perrey S, Denis R, Bishop D: Maximal power, but not fatigability, is greater during repeated sprints performed in the afternoon. Chronobiol. Int. 27(4), 855-864 (2010)

34. Scott TJ, Black CR, Quinn J, Coutts AJ: Validity and reliability of the session-RPE method for quantifying training in Australian football: a comparison of the CR10 and CR100 scales. J. Strength Cond. Res. 27(1), 270-276 (2013)

35. Teo W, Newton MJ, McGuigan MR: Circadian rhythms in exercise performance: implications for hormonal and muscular adaptation. J. Sports Sci. Med. 10, 600-606 (2011)

36. Thompson A, Jones H, Marqueze E, Gregson W, Atkinson G: The effects of evening bright light exposure on subsequent morning exercise performance. Int. J. Sports Med. 36(2), 101-106 (2015)

37. Wang $\mathrm{CH}, \mathrm{Wu} \mathrm{SB}, \mathrm{Wu} \mathrm{YT}$, Wei YH: Oxidative stress response elicited by mitochondrial dysfunction: implication in the pathophysiology of aging. Exp. Biol. Med. (Maywood) 238(5), 450-460 (2013) 